\title{
Quantum coherence enfeebled by classical uncertainties
}

\author{
R. O. Barrachina $\odot,{ }^{1,2,3,{ }^{*}}$ F. Navarrete $\odot,{ }^{4,5, \dagger}$ and M. F. Ciappina $\odot^{6,7,8,9, *}$ \\ ${ }^{1}$ Centro Atómico Bariloche, Comisión Nacional de Energía Atómica, Avenida Bustillo 9500, 8400 Bariloche, Argentina \\ ${ }^{2}$ Instituto Balseiro, Comisión Nacional de Energía Atómica and Universidad Nacional de Cuyo, \\ Avenida Bustillo 9500, 8400 Bariloche, Argentina \\ ${ }^{3}$ Consejo Nacional de Investigaciones Científicas y Técnicas (CONICET), Argentina \\ ${ }^{4}$ Department of Physics, Kansas State University, Manhattan, Kansas 66506, USA \\ ${ }^{5}$ Institute of Physics, University of Rostock, 18051 Rostock, Germany \\ ${ }^{6}$ ICFO-Institut de Ciencies Fotoniques, The Barcelona Institute of Science and Technology, 08860 Castelldefels (Barcelona) \\ ${ }^{7}$ Institute of Physics of the ASCR, ELI Beamlines Project, Na Slovance 2, 18221 Prague, Czech Republic \\ ${ }^{8}$ Physics Program, Guangdong Technion - Israel Institute of Technology, Shantou, Guangdong 515063, China \\ ${ }^{9}$ Technion - Israel Institute of Technology, Haifa 32000, Israel
}

(Received 9 June 2020; accepted 16 November 2020; published 10 December 2020)

\begin{abstract}
The fundamental indication of the departure of quantum mechanics from the classical world is the so-called quantum coherence. Typically, we define it as the characteristic of systems which are in a superposition of states yielding interference patterns in certain kinds of experiments. In addition to its captivating philosophical implications, quantum coherence turned out to be a valuable tool in different areas, ranging from quantum information to biology, where it was used to describe several fundamental processes. Here, we go one step further to study how classical uncertainties in a mixture of similar states reduce quantum coherence in quantum scattering theory. To this end, we deal with different examples, all of them with roots in the widely studied Feynman's two-slit thought experiment. We finally propose an operational and intuitive definition of the concept of coherence length whose implications largely transcend the simplicity of the corresponding mathematical development, as it is demonstrated when applied to the analysis of some recent atomic and molecular processes.
\end{abstract}

DOI: 10.1103/PhysRevResearch.2.043353

\section{INTRODUCTION}

Quantum coherence is the instrumental signature that makes the quantum world different from the classical one. Besides its philosophical implications, it has proven to be decisive in different areas, ranging from quantum information to biology. Quantum coherence is indeed a challenging concept to both learn and teach, which is not usually included in courses and textbooks on quantum mechanics. Except for a few exceptions [1-3], where this subject is treated briefly and superficially, it is difficult to find any reference to coherence in the index of the most well-known textbooks [4-12]. Some books $[1,13,14]$ describe the coherent state of a linear oscillator concerning electromagnetic fields [15]. But we must point out that this does not cover every aspect of the broad concept of quantum coherence, as we will show in this work, where we will analyze its role in quantum scattering of spinless, massive particles.

\footnotetext{
*barra@cab.cnea.gov.ar

†navarrete@phys.ksu.edu

${ }^{\star}$ marcelo.ciappina@gtiit.edu.cn
}

Published by the American Physical Society under the terms of the Creative Commons Attribution 4.0 International license. Further distribution of this work must maintain attribution to the author(s) and the published article's title, journal citation, and DOI.
It is not an overstatement to say that, for a complete understanding of scattering phenomena, the concept of coherence should be one of the cornerstones to be clearly understood by invoking quantum mechanics. In other words, one should have to be able to satisfactorily answer elemental questions as, for example, "what is the maximum separation between the slits for Feynman's two-slit thought experiment [16] to be successful?" or "how is it possible that in a collision experiment the projectile beam could be represented by a single plane wave?"(it is worth to remind the reader here that when one refers to a projectile beam in a quantum scattering experiment, in fact the particles are always assumed to be thrown one-byone to the target, like in Feynman's thought experiment and Rutherford's seminal experiment [17] of quantum scattering). But chances are that most of us would fail. When analyzing scattering processes, we simply lack the conceptual tools to build a coherent answer to practically any questions related to quantum coherence. Perhaps the main reason for this failure is that while the standard syllabus of most introductory courses only deals with pure quantum states; in real-world massive particle scattering-experiments, such as those involved in the two previous questions, a much more complex analysis is required.

In 1927, von Neumann [18] introduced the concept of "density operator" as a comprehensive way of incorporating classical statistics into the description of real quantum systems. As it is shown in this article, this formalism easily 
leads to an operational (i.e., measurable) definition of quantum coherence, while providing a simple demonstration of the theorem of Van Cittert and Zernike [19,20] applied to quantum scattering of massive particles, i.e., how coherence builds up with time.

For a coherent collection of single-particle collision events, its hallmark is given by its capacity to produce effects that cannot be described within the classical framework. Interference is the more noticeable and well known of these effects, as exemplified by the widely studied Feynman's two-slit thought experiment. Yet, for this setup to function, i.e., for an interference pattern to show up, the particle beam has to illuminate the two slits in a coherent way. To this end, we introduced a quantity, the so-called coherence length, such that for the interference to be distinguishable, it has to be larger than or at least comparable with the distance between the two slits. On the contrary, if the coherence length is much smaller than the distance between the slits, the beam will not be able to cover both slits at the same time, and the interference pattern will cease to exist [21].

Our way to quantify coherence through the coherence length in the current work is purely operational (or can be even seen as "ad hoc") compared to more well-established theories that quantify coherence for correlated systems and have been comprehensively developed through the past decades. Nevertheless, the simplicity of the systems for which the coherence length can be defined, allows us to explore Feynman's twoslit thought experiment in more detail, which configures a paradigmatic example in quantum scattering theory. We think the later is a small but qualitatively different contribution to the understanding and quantification of coherence as a broad subject.

Here it is worth to mention that, even though in this work we quantify coherence in scattering processes through the coherence length, our contribution should not be mistaken with other ones which gauge coherence as a resource [22], a concept which is central for quantum information theory, with entanglement being the most paradigmatic case. Furthermore, although scattering processes can also be studied in terms of entanglement [23], the ontology of our subject study is different to such investigations, as well as the way we quantify coherence via the coherence length concept. Indeed, our approach aims to contribute to the full understanding of the collision between a massive spinless particle and an unstructured target.

This article is organized as follows. In the following section, we revisit the well-known Feynman's two-slit thought experiment, and explore it thoughtfully in steps of increasing complexity. Let us point out that we manage to do so in simple analytical terms. First, we consider the case where the two-slit arrangement is reached by a plane wave. After exploring this simple and standard representation of the thought experiment, we analyze what changes when the two slits are not hit by a plane wave but by a wave packet of varying width and impact parameter. Finally, we move on to describing the more realistic situation when we do not have total control of this impact parameter, but can only assert a given probabilistic distribution for it as, for instance, is the case when employing a collimator for narrowing the width of an incoming beam of particles. At this point, we show how, for quantum scattering of massive particles in general, it is possible to give an operative definition of the coherence length and even get a simple analytical expression for it. Finally, we show how the classical uncertainties in the impact parameter of the pure states that make up the quantum mixture, lead not only to a decrease in coherence while, at the same time, to the appearance of an effect analogous to the van Cittert-Zernike effect but for quantum scattering of massive particles. It should be noted that while it was predictable that classical uncertainties would weaken the quantum coherence of a system in a mixed state, the appearance of the van Cittert-Zernike effect in such simple and understandable terms represents a remarkable bonus of the present approach [24].

\section{FEYNMAN'S TWO-SLIT THOUGHT EXPERIMENT REVISITED}

Let us consider the standard Feynman's two-slit thought experiment with particles [16]. It can be thought of as the typical setup used for instance by Rutherford in his famous work [17], i.e., the projectile beam is assumed to be prepared in a similar way, but replacing the target atom with two structureless neutral and spin-less target "slits." If the projectile beam was replaced by classical projectiles thrown one-by-one at the target one would not find an interference pattern in a particle detector behind the slits, while for quantum projectiles one would expect to find a pattern which would share similarities with that of Young's two-slit experiment [25] of light waves. Nevertheless, we must remind the reader that throughout the work we are only referring to massive particles, not photons. Furthermore, in this work we do not study beams of highdensity atomic, fermionic, or bosonic highly correlated gases [26] but, as was mentioned in the Introduction, to a beam of single-particle, noninteracting and nonoverlapping in space or time projectiles.

We model the slits' array by means of two Gaussian functions [27] of width $\delta$ separated by a distance $a$, namely,

$$
S(x)=S_{0}\left[G_{\delta}\left(x-\frac{a}{2}, 0\right)+G_{\delta}\left(x+\frac{a}{2}, 0\right)\right],
$$

where (see the Appendix for more details)

$G_{s}(x, L)=\left(\frac{2 s^{2}}{\pi\left(s^{2}+i \lambda L / \pi\right)^{2}}\right)^{1 / 4} \exp \left(-\frac{x^{2}}{s^{2}+i \lambda L / \pi}\right)$.

Here, $S_{0}$ defines a normalization constant that-for the time being_-plays no role in our analysis.

We are assuming that the distance $a$ between the slits is much larger than their width $\delta$, so that no spurious aperture would appear at the center of the two-slit arrangement

$$
\begin{aligned}
S^{2}(x)=S_{0}^{2} & {\left[G_{\delta}^{2}\left(x-\frac{a}{2}, 0\right)+G_{\delta}^{2}\left(x+\frac{a}{2}, 0\right)\right.} \\
& \left.+2 e^{-a^{2} / 2 \delta^{2}} G_{\delta}^{2}(x, 0)\right] \\
\approx S_{0}^{2} & {\left[G_{\delta}^{2}\left(x-\frac{a}{2}, 0\right)+G_{\delta}^{2}\left(x+\frac{a}{2}, 0\right)\right] . }
\end{aligned}
$$




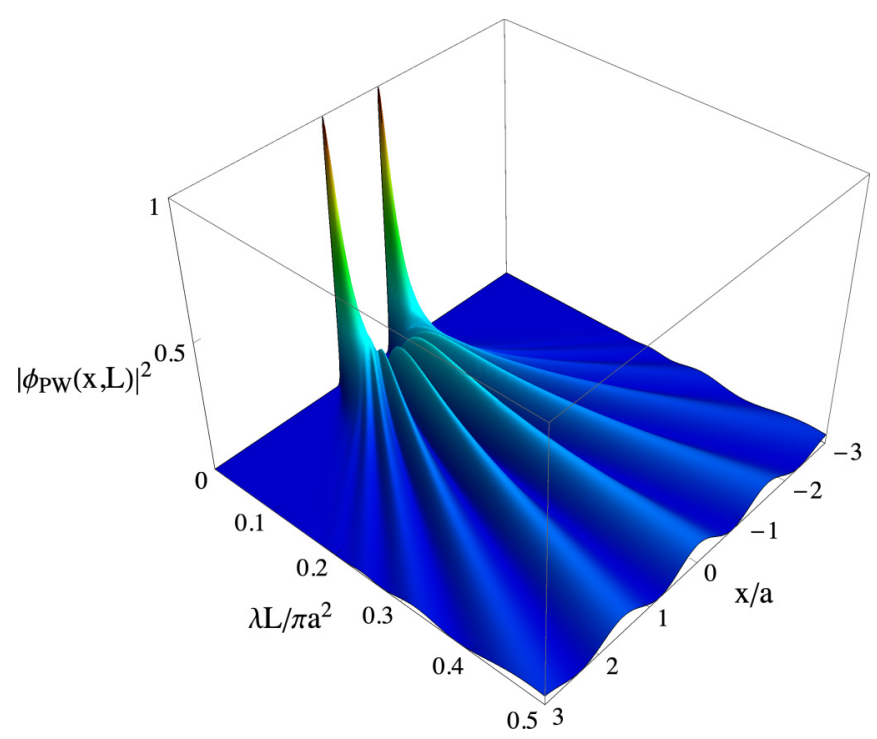

FIG. 1. Probability distribution $\left|\phi_{\mathrm{PW}}(x, L)\right|^{2}$ for a plane wave traversing two Gaussian slits of width $\delta$ separated by a distance $a=10 \delta$

To assure this condition, in all examples and figures shown throughout this article we will assume that $a=10 \delta$, for which $e^{-a^{2} / 2 \delta^{2}} \approx 2 \times 10^{-22}$.

At a first sight, representing the two slits using Eqs. (1) and (3) could appear unusual, although, as Feynman and Hibbs [27] pointed out, "we do not know how to design metal parts for our imaginary experiment which will produce such a gaussian slit. However, there is no conceptual difficulty."

Having defined our two-slit array, let us now bombard it with a different kind of projectiles, namely, plane waves, Gaussian wave packets, and mixed states.

\section{INCOMING PLANE WAVE}

We first consider a situation where the slits are reached by a plane wave traveling along a perpendicular direction $z$ with velocity $v$. This represents the standard version of Feynman's two-slit thought experiment, as presented in most textbooks on quantum mechanics. After passing through the slits, the wave function can be written analytically by means of two Gaussian wave packets [28]

$$
\phi_{\mathrm{PW}}(x, L)=S_{0} e^{i p L}\left[G_{\delta}\left(x-\frac{a}{2}, 0\right)+G_{\delta}\left(x+\frac{a}{2}, 0\right)\right] \text {, }
$$

where $\lambda=2 \pi \hbar / m v$ is the de Broglie wavelength and $L=v t$ is the distance traveled by the wave along $z$ in a time $t$.

In Fig. 1 the corresponding probability distribution

$$
\begin{aligned}
\left|\phi_{\mathrm{PW}}(x, L)\right|^{2}= & S_{0}^{2}\left\{\left|G_{\delta}\left(x-\frac{a}{2}, L\right)\right|^{2}+\left|G_{\delta}\left(x+\frac{a}{2}, L\right)\right|^{2}\right. \\
& \left.+2 \operatorname{Re}\left[G_{\delta}\left(x-\frac{a}{2}, L\right) G_{\delta}^{*}\left(x+\frac{a}{2}, L\right)\right]\right\},
\end{aligned}
$$

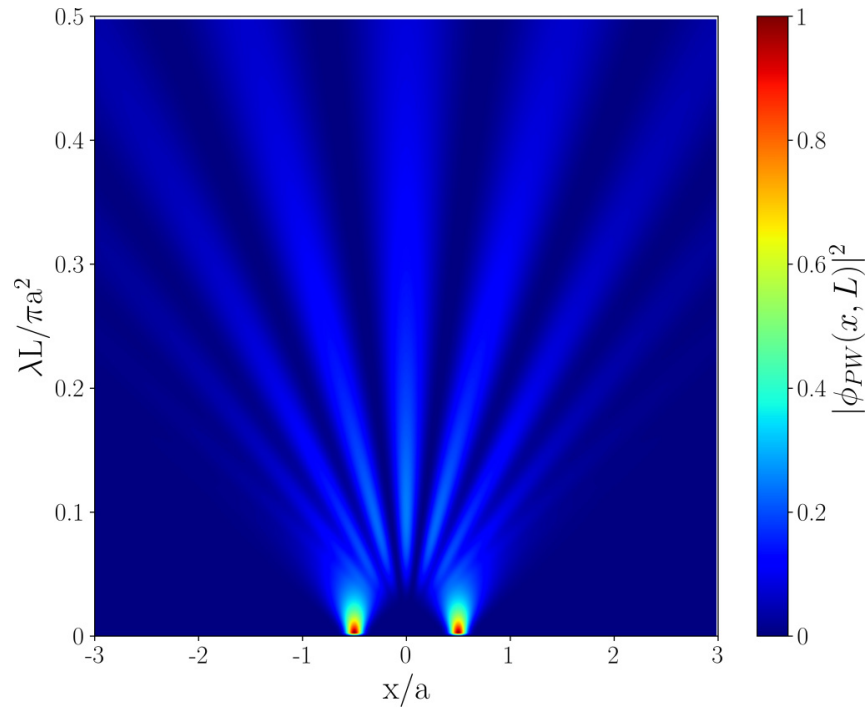

FIG. 2. $x y$ projection of Fig. 1 .

is depicted. As expected, it shows the standard Young interference structure.

Let us note that the width of each Gaussian wave packet in Eq. (1) grows with $L$ as [28]

$$
\tilde{\delta}=\left|\delta+i\left(\frac{\lambda L}{\pi \delta}\right)\right|=\sqrt{\delta^{2}+\left(\frac{\lambda L}{\pi \delta}\right)^{2}} .
$$

For small values of $L$, this width is much smaller than the distance between the slits $a$, and therefore, both wave packets spread independently without interfering with each other. On the other hand, for $\tilde{\delta}>a / 2$ (i.e., for $\lambda L / \pi a>\delta / 2$ ) the wave packets superimpose and the interference occurs. In particular, for even larger values of $L, \tilde{\delta}$ grows as $\lambda L / \pi \delta$, which explains the conical shape of the waves' spreading.

Finally, let us note that Eq. (5) can be written as

$$
\begin{aligned}
\left|\phi_{\mathrm{PW}}(x, L)\right|^{2}= & 2 S_{0}^{2}\left|G_{\delta}\left(\sqrt{x^{2}+\frac{a^{2}}{4}}, L\right)\right|^{2} \\
& \times\left[\cosh \left(\frac{2 a x}{\tilde{\delta}^{2}}\right)+\cos \left(\frac{2 \lambda \operatorname{Lax}}{\pi \delta^{2} \tilde{\delta}^{2}}\right)\right] .
\end{aligned}
$$

If the distance $L$ is large (i.e., in the far-field or Fraunhofer limit), this probability distribution can be approximated by

$$
\begin{aligned}
\left|\phi_{\mathrm{PW}}(x, L)\right|^{2} & \approx \sqrt{2 \pi} S_{0}^{2} \frac{2 \delta}{\lambda L}\left[1+\cos \left(\frac{2 \pi a x}{\lambda L}\right)\right] \\
& =\sqrt{2 \pi} S_{0}^{2} \frac{4 \delta}{\lambda L} \cos ^{2}\left(\frac{\pi a x}{\lambda L}\right),
\end{aligned}
$$

and, therefore, the spacing of the interference fringes is given by the standard expression $\lambda L / a$.

All these characteristics of the interference structure, namely the conical shape of the waves' spreading and the appearance of fringes for $\tilde{\delta}>a / 2$, with a spacing $\lambda L / a$ in the far-field limit, can be easily seen in Fig. 2, which shows a projection of Fig. 1 in the $x y$ plane. 


\section{INCOMING PURE GAUSSIAN STATE}

Let us now assume that the two-slit arrangement is reached by a pure Gaussian state $G_{d}(x-b, 0)$ of width $d$ and which is displaced by an impact parameter $b$ with respect to the center of the two slits, so that,

$$
\begin{aligned}
\phi_{b}(x, 0)= & S(x) G_{d}(x-b, 0) \\
= & S_{0}\left[G_{\delta}\left(x-\frac{a}{2}, 0\right)+G_{\delta}\left(x+\frac{a}{2}, 0\right)\right] G_{d}(x-b, 0) \\
= & S_{0}\left(\frac{2}{\pi \delta d}\right)^{1 / 2}\left[\exp \left(-\frac{\left(x-\tilde{a}_{+} / 2\right)^{2}}{\tilde{\sigma}_{o}^{2}}-\varphi_{+}\right)\right. \\
& \left.+\exp \left(-\frac{\left(x-\tilde{a}_{-} / 2\right)^{2}}{\tilde{\sigma}_{o}^{2}}-\varphi_{-}\right)\right]
\end{aligned}
$$

where

$$
\begin{gathered}
\tilde{a}_{ \pm}=\frac{2 b \delta^{2} \pm a d^{2}}{d^{2}+\delta^{2}}, \\
\tilde{\sigma}_{0}^{2}=\frac{d^{2} \delta^{2}}{d^{2}+\delta^{2}}, \\
\varphi_{ \pm}=\frac{(b \mp a / 2)^{2}}{d^{2}+\delta^{2}} .
\end{gathered}
$$

Note that in this case the slits are no longer located at $\pm a / 2$ but at $\tilde{a}_{ \pm} / 2$. The use of a Gaussian two-slit array in Eq. (1) simplifies all the calculations, but at the same time is responsible for this distortion of the slits positions. To avoid this artifact, we require that $d \gg \delta$. By applying this restriction, we can make Eq. (9) "separable," in the sense that the $x$-dependence is now restricted to the Gaussians of width $\delta$ exclusively,

$$
\begin{aligned}
\phi_{b}(x, 0) \approx & S_{0}\left[G_{\delta}\left(x-\frac{a}{2}, 0\right) G_{d}\left(b-\frac{a}{2}, 0\right)\right. \\
& \left.+G_{\delta}\left(x+\frac{a}{2}, 0\right) G_{d}\left(b+\frac{a}{2}, 0\right)\right] .
\end{aligned}
$$

Its time evolution can be simply obtained as

$$
\begin{aligned}
\phi_{b}(x, L) \approx & S_{0} e^{i p L}\left[G_{\delta}\left(x-\frac{a}{2}, L\right) G_{d}\left(b-\frac{a}{2}, 0\right)\right. \\
& \left.+G_{\delta}\left(x+\frac{a}{2}, L\right) G_{d}\left(b+\frac{a}{2}, 0\right)\right] .
\end{aligned}
$$

Note that, since

$$
\begin{aligned}
\left|\phi_{b}(x, L)\right|^{2} \approx & S_{0}^{2}\left\{\left|G_{\delta}\left(x-\frac{a}{2}, L\right) G_{d}\left(b-\frac{a}{2}, 0\right)\right|^{2}\right. \\
& +\left|G_{\delta}\left(x+\frac{a}{2}, L\right) G_{d}\left(b+\frac{a}{2}, 0\right)\right|^{2} \\
& +2 e^{-a^{2} / 2 d^{2}} \operatorname{Re}\left[G_{\delta}\left(x-\frac{a}{2}, L\right)\right. \\
& \left.\left.\times G_{\delta}^{*}\left(x+\frac{a}{2}, L\right)\right]\left|G_{d}(b, 0)\right|^{2}\right\}
\end{aligned}
$$

represents that part of the incoming wave that is transmitted through the filter $S(x)$, it must not be normalized. Actually, the transmission coefficient

$$
\mathcal{T}=\int_{-\infty}^{\infty}\left|\phi_{b}(x, L)\right|^{2} d x,
$$

depends on the impact parameter $b$. For instance, a large transmission is expected to occur for $b \approx \pm a / 2$, while it would approach zero for $b \rightarrow \pm \infty$. Thus, we decided to use the condition

$$
\lim _{L \rightarrow 0} \phi_{b}(x, L)=\phi_{b}(x, 0) .
$$

Before going on with the analysis of Eq. (15), let us rewrite it as follows:

$$
\begin{aligned}
\left|\phi_{b}(x, L)\right|^{2} \approx & 2 S_{0}^{2}\left|G_{\delta}\left(\sqrt{x^{2}+\frac{a^{2}}{4}}, L\right) G_{d}\left(\sqrt{b^{2}+\frac{a^{2}}{4}}, 0\right)\right|^{2} \\
\times & {\left[\cosh \left(\frac{2 a x}{\tilde{\delta}^{2}}+\frac{2 b a}{d^{2}}\right)+\cos \left(\frac{2 \lambda L a x}{\pi \delta^{2} \tilde{\delta}^{2}}\right)\right], }
\end{aligned}
$$

where $\tilde{\delta}$ was already defined in Eq. (6). Let us start by considering the case $b=0$. The resulting probability distribution

$$
\begin{aligned}
\left|\phi_{0}(x, L)\right|^{2} \approx & 2 S_{0}^{2}\left|G_{\delta}\left(\sqrt{x^{2}+\frac{a^{2}}{4}}, L\right) G_{d}\left(\frac{a}{2}, 0\right)\right|^{2} \\
& \times\left[\cosh \left(\frac{2 a x}{\tilde{\delta}^{2}}\right)+\cos \left(\frac{2 \lambda L a x}{\pi \delta^{2} \tilde{\delta}^{2}}\right)\right],
\end{aligned}
$$

is identical to $\left|\phi_{0}(x, L)\right|^{2}$ in Eq. (5), except for the presence of a multiplying factor

$$
G_{d}^{2}\left(\frac{a}{2}, 0\right)=\left(\frac{2}{\pi d^{2}}\right)^{1 / 2} e^{-a^{2} / 2 d^{2}} .
$$

When $d \gtrsim a$, the incoming wave "illuminates" both slits, and the interference structure might be clearly observed. On the other hand, for $d$ much smaller than $a$, only the edges of a comparatively narrow wave function transverse the slits, and the transmission drastically drops. For instance, due to the factor $G_{d}^{2}(a / 2,0)$, the probability distribution $\left|\phi_{0}(x, L)\right|^{2}$ for $d=a / 10$ is $3 \times 10^{22}$ smaller than for $d=a$, independently of $x$ and $L$. This means that the interference structure is still there, and is identical to that of a plane wave, but is too faint to be observed.

Now let us chose $b=-a / 2$ so as to locate the incoming pure state just on one of the slits. In Fig. 3 the probability $\left|\phi_{-a / 2}(x, L)\right|^{2}$ at a distance $L$ after the slits is shown. In clockwise order starting from the top left, the panels of Fig. 3 correspond to $d / a=10,1,0.75$, and 0.25 , respectively. They are associated to $e^{-a^{2} / 2 d^{2}} \approx 0.9950,0.6065,0,4111$, and 0,0003, respectively, in Eq. (15).

For $d=10 a$, the width of the incoming Gaussian state is so large in comparison with the distance between the slits that the result is practically identical to that of a plane wave. On the other hand, for $d=a$, the wave is mainly traversing one of the slits. The contribution of the other slit is much smaller, but still significant enough to produce a distinguishable interference structure. This ingredient is hardly discernible for $d=3 a / 4$, and is practically absent for $d=a / 4$. 


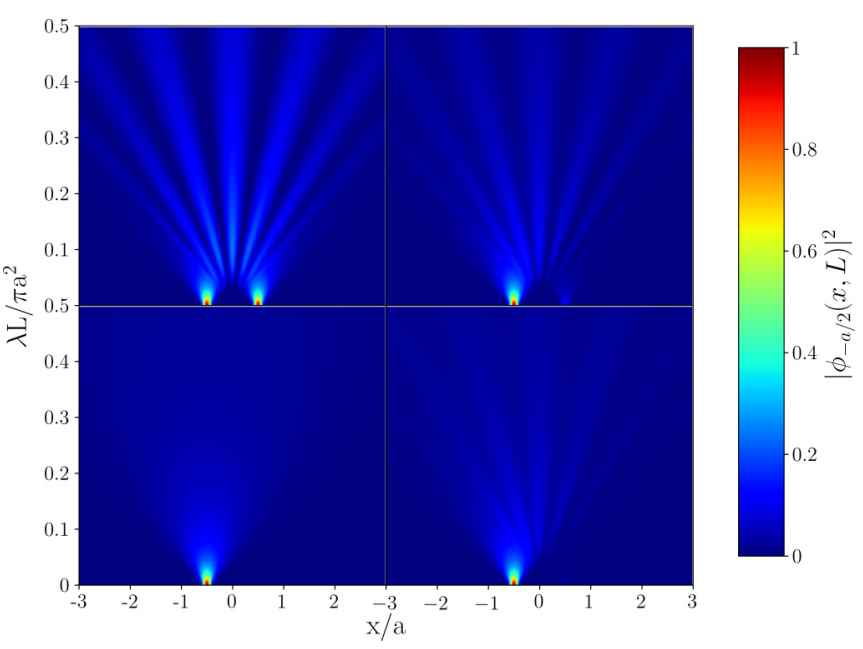

FIG. 3. Probability distribution for a Gaussian wave of width $d$ and impact parameter $b=-a / 2$ traversing two Gaussian slits of width $\delta$ separated by a distance $a=10 \delta$. In clockwise order starting from the top left, the panels correspond to $d / a=10,1,0.75$, and 0.25 , respectively.

This result demonstrates that even if the beam is purely coherent, the interference pattern might disappear if the width of the incoming wave functions is much smaller than the distance between the slits.

\section{INCOMING MIXED STATE}

In the previous section, the slits are reached by a collection of freely moving single-particles which do not overlap with each other either in space or time (i.e., single particles thrown one-by-one, like in Feynman's two-slit thought-experiment), all of which were described by the same quantum state $G_{d}(x-b, 0)$. We say that this collection is, by definition, purely coherent [29]. Note that we are considering an extremely restrictive condition on the collection to be purely coherent: we are assuming that it is feasible to produce a series of identical particles of mass $m$ with the same impact parameter $d$, the same amplitude, the same phase, and so on; in other words, the same quantum state. It should be clear that this is an abstraction, and that in any real situation some kind of deviation between the different quantum states should be unavoidable. Perhaps, it would be even more satisfactory from a pedagogical point of view to define a "purely" coherent collection by starting with a "partially" coherent state and then considering the two opposite limits of pure coherence and incoherence. But, since almost every textbook on quantum physics deals with purely coherent systems (in most cases without even acknowledging it), we also considered to be more easygoing to start from this particular limit, as shown in the previous section, and then move on towards the definition of "partially coherent" or "mixed" states.

As was argued earlier, the fingerprint of a coherent collection of single-particle collision events is its ability to produce effects that cannot be described in classical terms. The more conspicuous and well known of this effect is that of "interference," as exemplified by Feynman's two-slit thought experiment which, in Feynman's opinion [16]
... is impossible, absolutely impossible, to explain in any classical way, and which has in it the heart of quantum mechanics. In reality, it contains the only mystery. We cannot make the mystery go away by "explaining" how it works. We will just tell you how it works. In telling you how it works we will have told you about the basic peculiarities of all quantum mechanics.

Feynman was obviously aware that some subtleties would make the apparatus to be on an impossibly small scale to show the effects we are interested in; and warns the reader not try to set up this experiment. However, it is fair to say that at the time of the publication of Feynman's book, this supposedly thought experiment had already been done by Jönsson in 1961 [30]. This early experiment was later refined by Merli et al. [31] and Tonomura et al. [32]. Other realizations of this famous experiment were performed since then (see, for example, [33] and references therein). An atomic-size version of Feynman's two-slit thought experiment with electrons was finally performed in 2007 by Chesnel et al. [34-36].

The main issue with these experiments, both thought and real, is that the beam of electrons has to "illuminate" the two slits coherently for the interference effect to pop up. Surprisingly enough, even if the beam is purely coherent, that is if each and every electron in the beam can be described by the same wave function; even then the previous condition can fail. This is what we demonstrated in the previous section. And the reason for that is very simple: for the interference to be observable, the "full" spatial width of the wave function has to be larger than the distance between the slits, for allowing the formation of an observable interference pattern.

Let us now consider the more realistic case of a mixed state composed of wave functions $\phi_{b}(x, L)$, as in the previous section, but where $b$ is distributed according to a given classical distribution, namely,

$$
\rho_{0}(x, L)=\int d b G_{D}^{2}\left(b-b_{0}, 0\right)\left|\phi_{b}(x, L)\right|^{2} .
$$

For the classical probability we chose a Gaussian distribution of width $D$ and centered in some impact parameter $b_{0}$. As it is described in the Appendix, this integral can be easily computed

$$
\begin{aligned}
\rho_{0}(x, L)= & S_{0}^{2}\left\{\left|G_{\delta}\left(x-\frac{a}{2}, L\right) G_{\sqrt{d^{2}+D^{2}}}\left(b_{0}-\frac{a}{2}, 0\right)\right|^{2}\right. \\
& +\left|G_{\delta}\left(x+\frac{a}{2}, L\right) G_{\sqrt{d^{2}+D^{2}}}\left(b_{0}+\frac{a}{2}, 0\right)\right|^{2} \\
& +2 e^{-a^{2} / 2 d^{2}} \operatorname{Re}\left[G_{\delta}\left(x-\frac{a}{2}, L\right) G_{\delta}^{*}\left(x+\frac{a}{2}, L\right)\right] \\
& \left.\times\left|G_{\sqrt{d^{2}+D^{2}}}\left(b_{0}, 0\right)\right|^{2}\right\} .
\end{aligned}
$$

Note that for $D \rightarrow 0$, the Gaussian distribution $G_{D}^{2}\left(b-b_{0}, 0\right)$ in Eq. (21) becomes a representation of the Dirac delta function $\delta\left(b-b_{0}\right)$, and therefore, $\rho_{0}(x, L)$ equals $\left|\phi_{b_{0}}(x, L)\right|^{2}$. However, since $D$ is usually a macroscopic length associated to the collimator in an actual realization of Feynman's two-slit thought experiment, while $d$ is of atomic dimensions, in the rest of the article we will safely assume that $D \gg d$. In this 


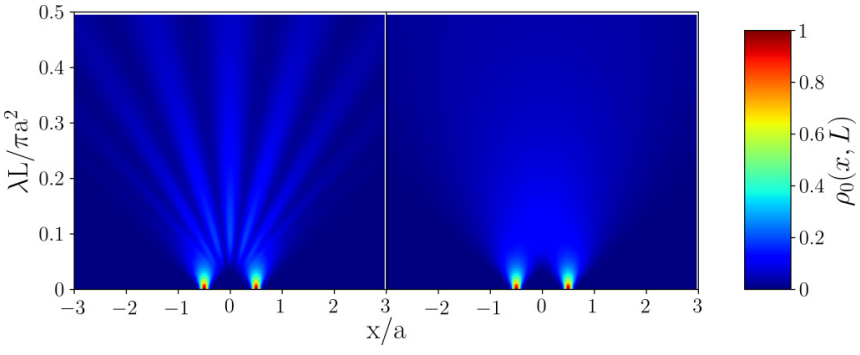

FIG. 4. Probability distribution $\rho_{0}(x, L)$ for mixed states traversing two Gaussian slits of width $\delta$ separated by a distance $a=10 \delta$ for $D=10 a$. The left and right panels correspond to $d=a$ and $d=a / 10$, respectively.

same line of thought, and without loss of generality, we will also assume that $b_{0}=0$. Thus, we obtain

$$
\begin{aligned}
\rho_{0}(x, L)= & S_{0}^{2}\left\{\left|G_{\delta}\left(x-\frac{a}{2}, L\right) G_{D}\left(\frac{a}{2}, 0\right)\right|^{2}\right. \\
& +\left|G_{\delta}\left(x+\frac{a}{2}, L\right) G_{D}\left(\frac{a}{2}, 0\right)\right|^{2} \\
& +2 e^{-a^{2} / 2 d^{2}} \operatorname{Re}\left[G_{\delta}\left(x-\frac{a}{2}, L\right) G_{\delta}^{*}\left(x+\frac{a}{2}, L\right)\right] \\
& \left.\times\left|G_{D}(0,0)\right|^{2}\right\},
\end{aligned}
$$

which again is similar to $\left|\phi_{b_{0}}(x, L)\right|^{2}$, but with the width $d$ replaced by $D$, except for the factor $e^{-a^{2} / 2 d^{2}}$, which remains unchanged. This means that $D$ does not seem to play any significant role in the appearance or not of the interference effect. This is again controlled by the width $d$ of each individual wave function. Let us see this with an example. In Fig. 4 we plot the probability distribution $\rho_{0}(x, L)$ for mixed states traversing two Gaussian slits of width $\delta$ separated by a distance $a=10 \delta$ for $D=10 a$. The left and right panels correspond to $d=a$ and $d=a / 10$, respectively.

Note that in the right panel, for $d=a / 10$, each wave in the ensemble might at the most traverse one slit, but not both; and therefore it is incapable of producing any distinguishable interference structure. On the other hand, in the left panel, the width $d$ of each wave in the mixed state is of the order of the separation $a$ between the slits. Thus, some wave functions in the mixed states, namely those with small values of the impact parameter $b$, can hit both slits, and the result is practically identical to that of a plane wave. In other words, the appearance or disappearance of the interference pattern depends on whether the width $d$ of each wave in the mixed state is larger (or equal), or much smaller than the distance between the slits, respectively.

\section{VAN CITTERT-ZERNIKE EFFECT}

In the previous section, we saw that under particular circumstances, the interference structure disappears. As it was just explained, this occurs whenever the width $d$ of the incoming states is much smaller than the two-slit separation $a$. Now, let us consider the case when this same wave is allowed to travel a given distance $L_{0}$ before reaching the two-slit array. In this case, the initial Gaussian state $G_{d}(x-b, 0)$ should be replaced by $G_{d}\left(x-b, L_{0}\right)$, so that

$$
\rho_{L_{0}}(x, L)=\int d b G_{D}^{2}(b, 0)\left|\tilde{\phi}_{b}(x, L)\right|^{2},
$$

with

$$
\begin{aligned}
\tilde{\phi}_{b}(x, L)= & S_{0} e^{i p L}\left[G_{\delta}\left(x-\frac{a}{2}, L\right) G_{d}\left(b-\frac{a}{2}, L_{0}\right)\right. \\
& \left.+G_{\delta}\left(x+\frac{a}{2}, L\right) G_{d}\left(b+\frac{a}{2}, L_{0}\right)\right] .
\end{aligned}
$$

If we blindly rely on the results of the previous section, we could easily assume that the appearance or not of interference might depend on the relationship between the width

$$
\tilde{d}=\sqrt{d^{2}+\left(\frac{\lambda L_{0}}{\pi d}\right)^{2}},
$$

of each individual wave $G_{d}\left(x-b, L_{0}\right)$ when reaching the slits, and the separation $a$ between these slits. In fact, this is what we observed in all the cases discussed up to this point. However, if we actually perform the integral on $b$ by using the formulas in the Appendix, we obtain

$$
\begin{aligned}
\rho_{L_{0}}(x, L)= & S_{0}^{2}\left\{\left|G_{\delta}\left(x-\frac{a}{2}, L\right) G_{D}\left(\frac{a}{2}, \frac{D L_{0}}{d}\right)\right|^{2}\right. \\
+ & \left|G_{\delta}\left(x+\frac{a}{2}, L\right) G_{D}\left(\frac{a}{2}, \frac{D L_{0}}{d}\right)\right|^{2} \\
+ & 2 e^{-a^{2} / 2 \ell^{2}} \operatorname{Re}\left[G_{\delta}\left(x-\frac{a}{2}, L\right) G_{\delta}^{*}\left(x+\frac{a}{2}, L\right)\right] \\
& \left.\times\left|G_{D}\left(0, \frac{D L_{0}}{d}\right)\right|^{2}\right\},
\end{aligned}
$$

with

$$
\ell=\sqrt{d^{2}+\left(\frac{\lambda L_{0}}{\pi D}\right)^{2}},
$$

and the result is not the one we assumed. Instead, we find that the interference occurs, not when the width $\tilde{d}$ of each individual wave function is larger or at least comparable with $a$, but whenever the characteristic length is. The difference between $\tilde{d}$ and $\ell$ seems to be almost insignificant since it only amounts to replacing $d$ by $D$ in the denominator of the second term, although a closer look demonstrates that it is extremely relevant.

We will call this quantity $\ell$ the coherence length of the collection of scattering events (along the arbitrary direction $x$ ). It is clear that it provides a measurement of the coherence of the quantum system under consideration. Of course, this definition depends on multiple factors. For instance, depending on the detector, it should be possible to actually observe an interference pattern for slits separations larger than $\ell$. Thus, the above definition of $\ell$ has a certain degree of vagueness and arbitrariness that, on the other hand, is inevitable. Perhaps, it would be more appropriate to multiply it by a factor that might depend on the experimental setup, geometry, and so on. However, these subtleties do not affect the development 


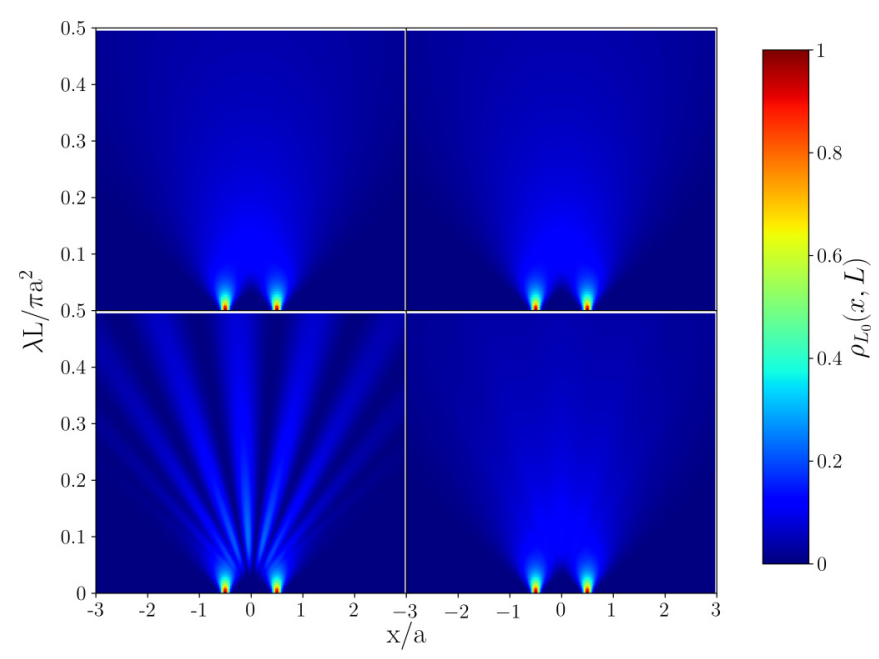

FIG. 5. Probability distribution $\rho_{L_{0}}(x, L)$ for mixed states traversing two Gaussian slits of width $\delta$ separated by a distance $a=10 \delta$ for $D=10 a$ and $d=a / 10$, for different values of $L_{0}$. In clockwise order starting from the top left, the panels correspond to $\lambda L_{0} / \pi a^{2}=0,0.1,4$, and 10 , respectively.

and conclusions of the present article, and therefore we will maintain the previous definition.

Let us first note that, as we stated in a previous section, in Eq. (27) we assumed that the classical uncertainty $D$ (as related to the collimator's characteristic dimensions) is much larger than the quantum mechanical uncertainty $d$ of each wave function in the collection and that $b_{0}=0$. We made these assumptions for the sake of simplicity, even though the general case for arbitrary values of $D$ and $b_{0}$ can be analytically evaluated.

Now, while in the Fresnel limit (i.e., for $L_{0} \approx 0$ ), we recover the same results of the previous section, where $D$ plays a minimal role, the appearance of this classical uncertainty becomes strongly dominant for very large values of $L_{0}$ (Fraunhofer limit), namely,

$$
\ell \approx \frac{\lambda L_{0}}{\pi D}
$$

Actually, if we compare this expression with that of the individual wave functions, $\tilde{d} \approx \lambda L_{0} / \pi d$, we immediately notice that the presence of the classical uncertainty $D$ dramatically reduces the coherence of the quantum system by a factor $d / D$. This result represents indeed the Van Cittert-Zernike effect of Optics applied to quantum particles, and gives meaning to the title of the present work, by showing that the classical uncertainty, characterized by the aperture $D$ of the collimator, reduces the quantum uncertainty, which is represented by $\ell$ instead of $\tilde{d}$.

This result is exemplified in Fig. 5, where we consider a beam of wave packets traversing a collimator and traveling a distance $L_{0}$ until reaching a two-slit arrangement of width $\delta$ separated by a distance $a=10 \delta$. The initial width of the wave packets and that of the collimator are $d=a / 10$ and $D=10 a$, respectively. In clockwise order starting from the top left, the panels correspond to $\lambda L_{0} / \pi a^{2}=0,0.1,4$, and 10 , respectively. In the first case, the coherence length $\ell$ is much smaller than the distance $a$ between the slits, and no interference structure is formed. This situation is identical to that of the right-side panel in Fig. 4. In the second panel in clockwise order $\lambda L_{0} / \pi a^{2}=d / a$. If the naive analysis before the Eq. (27) were correct (which it is not), the full interference structure should already be seen. But this does not happen. The reason, as it was already explained, is that even though the width $\tilde{d}$ of each wave is already equal to the separation $a$ between the slits, the classical uncertainty $D$ enfeebles the coherence length $\ell$ by a factor $d / D$ and, since it is much smaller than $a$, the interference does not occur. In the third panel in clockwise order, corresponding to a case where $\ell \approx 0.4 a$, a barely discernible interference structure might be observed. Finally, in the fourth panel, $\ell$ is equal to the width $a$ between the slits, and the interference fringes are fully developed.

Let us point out that since the coherence length $\ell$, Eq. (28), depends on the momentum of the incident particles only through its wavelength $\lambda$, it is similar to what would be expected in the case of electromagnetic waves. Thus, our version of Feynman's thought experiment is identical to the demonstration proposed by Thomas Young in 1807 [25], and is not different from the techniques used to measure the coherence of sunlight on Earth. However, since both the diameter $D$ of the Sun and its distance $L_{0}$ to the Earth is obviously fixed, what is varied in these experiments (see, e.g., [37]) is the distance $a$ between the slits, observing that the interference disappears when $a$ is greater than a coherence length $\ell$ of some few tens of nanometers.

Let us finally consider the case of a collision experiment. It has been usually assumed that the wavelength $\lambda$ of the incoming projectiles' beam (i.e., electrons, positrons, ions, atoms, or molecules), and the macroscopic collimator's width $D$ and flight distance $L_{0}$ in the accelerator and focusing section of standard equipments, are such that the corresponding coherence length $\ell$ is always much larger than the size $a$ of the atoms or molecules in the target chamber. Therefore, it has always been assumed that when reaching the target, the projectile's mixed state can be represented by a single plane wave. However, some recent experiments [38] showed that this is not always the case (see, e.g., [39] and references therein). Actually, by changing the distance $L_{0}$ and the width $D$ in the experimental setup, it has been possible to manipulate the coherence length $\ell$ between a mixed $(\ell<a)$ and a coherent situation $(\ell \geqslant a)$, as shown in the first and fourth panels of Fig. 5. These results have put into question a basic assumption of the standard scattering theory (namely that $\ell \gg a$ ), and asked for new experimental and theoretical approaches $[40,41]$ to the analysis of collision processes. We will not explore this phenomenon any further in this article. For a far more comprehensive theoretical discussion, we refer the reader to some recent publications $[42,43]$.

\section{CONCLUSION}

In this work, we performed a formal analysis on how to incorporate concepts of quantum coherence in scattering processes employing the coherence length. Its definition allowed us to both describe qualitatively and also quantify the influence of the classical experimental context in the observation of quantum mechanical effects, through a detailed analysis of Feynman's two-slit thought experiment, which is a central 
example in quantum scattering theory. We thoughtfully explored the problem in steps of increasing complexity, all of them in simple analytical terms, which is useful for both expanding the understanding of the subject as well as contributing to its pedagogy.

First, with respect to the advance in pedagogical grounds made by this work, we expect that, based on the concepts and notions provided in this contribution, a reader would be now in the position to respond coherently the basic questions like what is the maximum separation between the slits for Feynman's two-slit thought experiment with particles to be successful [of the order of the coherence length $\ell$ in Eq. (28)], or when is it possible to represent the projectile beam by a single plane wave in a collision experiment (which will be a suitable description when the coherence length is larger than the size of the atomic or molecular targets).

Finally, regarding our contribution to the benefits of the subject itself, we have to mention that we demonstrated how, for a mixed state, the classical uncertainties in the impact parameter of its pure state constituents lead to a decrease of the coherence and the appearance of an effect similar to the van Cittert-Zernike effect, but for the quantum scattering of massive particles. It is worth to notice that, while it was predictable that classical uncertainties would wash out quantum effects of a system in a mixed state, the appearance of this effect in such simple and understandable fashion is a clear advantage of the present theoretical development [24].

\section{ACKNOWLEDGMENTS}

This work was supported by Universidad Nacional de Cuyo (Grant No. 06/C550), the Spanish Ministry MINECO (National Plan 15 Grant: FISICATEAMO No. FIS2016-79508-P, SEVERO OCHOA No. SEV-2015-0522, FPI), European Social Fund, Fundació Cellex, Generalitat de Catalunya (AGAUR Grant No. 2017 SGR 1341, CERCA/Program), ERC AdG NOQIA, EU FEDER, the National Science Centre, Poland-Symfonia Grant No. 2016/20/W/ST4/00314 and the Grantová agentura České Re- publiky (GAČR Grant 20-24805J). F.N. acknowledges the hospitality of the James R. Macdonald Laboratory, Department of Physics, Kansas State University, Manhattan, Kansas, USA.

\section{APPENDIX}

Let us consider the Gaussian wave function

$$
G_{s}(x, L)=\left(\frac{2 s^{2}}{\pi\left(s^{2}+i \lambda L / \pi\right)^{2}}\right)^{1 / 4} e^{-x^{2} /\left(s^{2}+i \lambda L / \pi\right)} .
$$

The corresponding probability reads

$$
\left|G_{s}(x, L)\right|^{2}=\left(\frac{2}{\pi\left(s^{2}+(\lambda L / \pi s)^{2}\right)}\right)^{1 / 2} e^{-2 x^{2} /\left[s^{2}+(\lambda L / \pi s)^{2}\right]} .
$$

Some simple properties are

$$
G_{s}^{*}(x, L)=G_{s}(x,-L) \quad \text { and } \quad G_{s}(-x, L)=G_{s}(x, L) .
$$

On the other hand, the integral of a product of an arbitrary number of them reads

$$
\int_{-\infty}^{\infty} \prod_{n=1}^{N} G_{s_{n}}\left(x-a_{n}, L_{n}\right) d x=\sqrt{\pi} \Sigma e^{(\Sigma / h)^{2}} \prod_{n=1}^{N} G_{s_{n}}\left(a_{n}, L_{n}\right),
$$

with

$$
\frac{1}{\Sigma^{2}}=\sum_{n=1}^{N} \frac{1}{s_{n}^{2}+i \lambda L_{n} / \pi} \quad \text { and } \quad \frac{1}{h}=\sum_{n=1}^{N} \frac{a_{n}}{s_{n}^{2}+i \lambda L_{n} / \pi} .
$$

In particular,

$$
\int_{-\infty}^{\infty}\left|G_{s}(x-a, L)\right|^{2} d x=1
$$

as expected.
[1] A. Galindo and P. Pascual, Quantum Mechanics I (SpringerVerlag, Berlin, 1990), Chap. 2.

[2] C. Cohen-Tannoudji, B. Diu, and F. Laloë, Quantum Mechanics (2 vol. set) (John Wiley and Sons, Chichester, NH, 2006).

[3] R. G. Newton, Quantum Physics, A Text for Graduate Students (Springer, New York, 2002).

[4] L. I. Schiff, Quantum Mechanics, 3rd ed. (McGraw-Hill, New York, 1968), Chap. 10.

[5] J. J. Sakurai, Modern Quantum Mechanics (Addison-Wesley, Reading, MA, 1994).

[6] D. J. Griffiths, Introduction to Quantum Mechanics (Prentice Hall, Upper Saddle River, NJ, 1995).

[7] B. H. Bransden and C. J. Joachain, Quantum Mechanics, 2nd ed. (Pearson, Harlow, England, 2000), Chap. 14.

[8] J. S. Townsend, A Modern Approach to Quantum Mechanics (University Science Books, Sausalito, CA, 2000).
[9] W. Greiner, Quantum Mechanics, An Introduction (SpringerVerlag, Berlin, 2001).

[10] A. I. M. Rae, Quantum Mechanics (Institute of Physics, Bristol, England, 2002).

[11] A. C. Phillips, Introduction to Quantum Mechanics (Wiley, Chichester, NH, 2003).

[12] A. Messiah, Quantum Mechanics (Dover, Mineola, NY, 2014).

[13] L. D. Landau and E. M. Lifshitz, Quantum Mechanics, Non relativistic Theory (Butterworth-Heinemann, Amsterdam, 1981).

[14] S. Gasiorowicz, Quantum Physics, 3rd ed. (John Wiley, Hoboken, NJ, 2003), Suppl. 20-c.

[15] L. E. Ballentine, Quantum Mechanics, A Modern Development (World Scientific, Singapore, 2000).

[16] R. Feynman, R. Leighton, and M. Sands, The Feynman Lectures on Physics, Vol 3. Quantum Mechanics (Addison-Wesley, Reading, MA, 1971). 
[17] H. Geiger and E. Marsden, The laws of deflexion of a particles through large angles, The London, Edinb. Dublin Philos. Mag. J. Sci. 25, 604 (1913).

[18] J. von Neumann, Wahrscheinlichkeitstheoretischer Aufbau der Quantenmechanik, Göttinger Nachrichten 1927, 245 (1927).

[19] P. H. van Cittert, Die Wahrscheinliche Schwingungsverteilung in Einer von Einer Lichtquelle Direkt Oder Mittels Einer Linse Beleuchteten Ebene, Physica 1, 201 (1934).

[20] F. Zernike, The concept of degree of coherence and its application to optical problems, Physica 5, 785 (1938).

[21] R. O. Barrachina, F. Navarrete, and M. F. Ciappina, Free evolution of a mixture of quantum states with classical space and momentum uncertainties, Eur. J. Phys. 40, 065402 (2019).

[22] T. Baumgratz, M. Cramer, and M. B. Plenio, Quantifying Coherence, Phys. Rev. Lett. 113, 140401 (2014).

[23] M. Lamehi-Rachti and W. Mittig, Quantum mechanics and hidden variables: A test of Bell's inequality by the measurement of the spin correlation in low-energy proton-proton scattering, Phys. Rev. D 14, 2543 (1976).

[24] I. Fabre, F. Navarrete, L. Sarkadi, and R. O. Barrachina, Free evolution of an incoherent mixture of states: A quantum mechanical approach to the van Cittert-Zernike theorem, Eur. J. Phys. 39, 015401 (2018).

[25] T. Young, A Course of Lectures on Natural Philosophy and the Mechanical Arts (J. Johnson, London, 1807).

[26] K. E. Cahill and R. J. Glauber, Density operators for fermions, Phys. Rev. A 59, 1538 (1999).

[27] R. P. Feynman and A. R. Hibbs, Quantum Mechanics and Path Integrals (McGraw-Hill, New York, 1965).

[28] E. J. Heller, Time-dependent approach to semiclassical dynamics, J. Chem. Phys. 62, 1544 (1975).

[29] K. Blum, Density Matrix Theory and Applications (SpringerVerlag, Berlin, 2012).

[30] C. Jönsson, Elektroneninterferenzen an mehreren künstlich hergestellten Feinspalten, Z. Physik 161, 454 (1961); English translation: Electron diffraction at multiple slits, Am. J. Phys. 42, 4 (1974).

[31] P. G. Merli, G. F. Missiroli, and G. Pozzi, On the statistical aspect of electron interference phenomena, Am. J. Phys. 44, 306 (1976).
[32] A. Tonomura, J. Endo, T. Matsuda, T. Kawasaki, and H. Ezawa, Demonstration of single-electron buildup of an interference pattern, Am. J. Phys. 57, 117 (1989).

[33] H. Yanagisawa, M. F. Ciappina, C. Hafner, J. Schötz, J. Osterwalder, and M. F. Kling, Optical control of young's type double-slit interferometers for laser-induced electron emission from a nano-tip, Sci. Rep. 7, 12661 (2017).

[34] J.-Y. Chesnel, A. Hajaji, R. O. Barrachina, and F. Frémont, Young-Type Experiment Using a Single-Electron Source and an Independent Atomic-Size Two-Center Interferometer, Phys. Rev. Lett. 98, 100403 (2007).

[35] R. O. Barrachina and M. Zitnik, Young's interference effect in the autoionization of atoms colliding with molecules, J. Phys. B 37, 3847 (2004).

[36] R. O. Barrachina, Young electron interference effects in atomic ionization collisions, Rad. Phys. Chem. 76, 375 (2007).

[37] S. Divitt and L. Novotny, Spatial coherence of sunlight and its implications for light management in photovoltaics, Optica 2, 95 (2015).

[38] K. N. Egodapitiya, S. Sharma, A. Hasan, A. C. Laforge, D. H. Madison, R. Moshammer, and M. Schulz, Manipulating Atomic Fragmentation Processes by Controlling the Projectile Coherence, Phys. Rev. Lett. 106, 153202 (2011).

[39] M. Schulz, The role of projectile coherence in the few-body dynamics of simple atomic systems, Adv. Atom. Mol. Opt. Phys. 66, 507 (2017).

[40] L. Sarkadi, I. Fabre, F. Navarrete, and R. O. Barrachina, Loss of wave-packet coherence in ion-atom collisions, Phys. Rev. A 93 , 032702 (2016).

[41] F. Navarrete, M. F. Ciappina, L. Sarkadi, and R. O. Barrachina, The role of the wave packet coherence on the ionization cross section of $\mathrm{He}$ by $\mathrm{p}^{+}$and $\mathrm{C}^{6+}$ projectiles, Nucl. Instr. Meth. B 408, 165 (2017).

[42] R. O. Barrachina, F. Navarrete, M. F. Ciappina, and Michael Schulz, Coherence and contextuality in scattering experiments, in Ion-Atom Collisions: The Few-Body Problem in Dynamic Systems, edited by M. Schulz (De Gruyter, Berlin, 2019), pp. 61-79.

[43] F. Navarrete, R. O. Barrachina, and M. F. Ciappina, Distortion of the ionization cross section of he by the coherence properties of a $\mathrm{C}^{6+}$ beam, Atoms 7, 31 (2019). 